\title{
Adoption of Improved Practices Promoted under Vegetable Development Programme among the Tribals
}

\author{
Sonali Sharma ${ }^{1 *}$, Snehlata Maheshwari ${ }^{2}$, Rajshree Upadhyay ${ }^{2}$, \\ Bhupendra Upadhyay ${ }^{3}$ and B.R. Morwal ${ }^{1}$ \\ ${ }^{1}$ SMS (Home Science), KVK-Barmer (Raj.), India \\ ${ }^{2}$ Department of Home Science Extension and Communication Management, College of Home \\ Science MPUAT, Udaipur, (Raj.), India \\ ${ }^{3}$ Department of Statistics, Rajasthan College of Agriculture, MPUAT, Udaipur, (Raj.), India \\ *Corresponding author
}

\section{A B S T R A C T}

\begin{tabular}{|c|c|}
\hline & Vegetables make a major portion of human diet. Though the vegetable requirement is \\
\hline Keywords & $\begin{array}{l}\text { of vegetables in India have introduced. However, a planned development in a field of } \\
\text { vegetable production will not only improve the nutritional requirement for masses but can }\end{array}$ \\
\hline $\begin{array}{l}\text { Adoption, } \\
\text { Vegetable } \\
\text { development } \\
\text { programme }\end{array}$ & $\begin{array}{l}\text { also meet the challenge of adequate food supply to the growing population in India. } \\
\text { Vegetable has become highly commercialized. And in such competitive market it's very } \\
\text { essential to have knowledge and proper adoption of package of practices of vegetables. } \\
\text { Vegetable Development Programme under Agriculture services of Tribal Area }\end{array}$ \\
\hline Article Info & Development Programme focuses the same. To find out adoption of Tribals in the \\
\hline $\begin{array}{l}\text { Accepted: } \\
\text { 22 July } 2018 \\
\text { Available Online: } \\
10 \text { August } 2018\end{array}$ & $\begin{array}{l}\text { Development Programme among the tribals" was undertaken. The study was conducted in } \\
\text { four villages of lasadiya block of Udaipur district, covering } 60 \text { tribal farmers ( } 30 \\
\text { beneficiaries and } 30 \text { non-beneficiaries). The data were collected through pre-tested } \\
\text { structured interview schedule and appropriate statistical procedures were employed to }\end{array}$ \\
\hline & $\begin{array}{l}\text { analyze data. Investigation was made relating to adoption association with the improved } \\
\text { agriculture practices of onion, okra, tomato and brinjal. }\end{array}$ \\
\hline
\end{tabular}

\section{Introduction}

From time immemorial, agriculture has been a way of life and it is the principal source of livelihood for more than 55 per cent of the population of this country. Evolved in Africa and then Asia, we ate a huge range of leaves, buds, flower buds, stems, gums, roots, tubers, and even pollens. The number of plant families we used as food was very much greater than the restricted range we eat today. Wild foods were carefully selected to avoid the plants or parts of plants with bitter and unpleasant taste, which likely contained toxic compounds. Today's plants are more palatable, and yet paradoxically, we eat very few plants as part of our daily diet. The major contribution of plants to human health has always been thought to be the large amounts 
of vitamin $\mathrm{A}$, the folic acid vitamin, and the vitamin $\mathrm{C}$ they contained; as well as good amount of some minerals. It is becoming more and more obvious that there are many plant chemicals that act together to protect the human body from the onset of cancers and heart diseases, and that vitamin supplements can be helpful, but are not as useful as whole plant.

India, with its wide variability of climate and soil, has good potential for growing a wide range of horticultural crops such as fruits, vegetables, potato, tropical tuber crops and mushrooms, ornamental crops, medicinal and aromatic plants, species and plantation crops like coconut, cashew nut, cocoa, etc. Since the mid-eighties, the government identified horticulture crops as a means of diversification for making agriculture more profitable through efficient land use, optimum utilization of natural resources and creating skilled employment for rural masses and tribals with the past efforts rewarding.

More than 40 kinds of vegetables belonging to different groups, solonaceous, cucurbitaceous, leguminuous, cruciferous (cole crops) root crops and leafy vegetables are grown in India in tropical, sub-tropical and temperate regions. Important vegetable crops grown in the country are tomato, onion, brinjal, okra, cabbage etc. India is next only to china in area and production of vegetables. India contributes about $13 \%$ to the world vegetable production and occupies first position in the production off cauliflower, second in onion and third in the cabbage in the world.

For the growth of vegetable production, it is quiet essential to involved more farmers with technical knowledge and package of practices. Tribals are doing much well through various government developmental programmes and its adopting the technical knowledge with their agriculture knowledge. The term adoption in this study implied the acceptance and use of improved practices promoted under agricultural services of TADP by the beneficiaries of the service and nonbeneficiaries respondents.

\section{Materials and Methods}

The study was conducted in Udaipur district of Rajasthan as for the development of tribals a separate department i.e. Tribal Area Development Department is in function. The department implements various schemes or programmes for tribal development along with agricultural services. Vegetable Development programme was implemented in Lasadiya block of Udaipur district. Two villages were selected randomly for the beneficiaries from programme implementing villages and similarly two villages were selected for nonbeneficiaries from non programme villages. Thus there were 2 villages for beneficiaries and 2 villages for non-beneficiaries for the six agricultural services. Thus in total 4 villages were selected for the study purpose.

For selection of sample, thirty beneficiaries were selected randomly, for assessment of adoption a comparable matching sample of 30 non-beneficiary tribals was selected randomly. Thus the total sample for the study comprised of 60 respondents. The scoring of the schedule was finalized in consultation with the researcher's advisory committee members. The scoring in part of the interview schedule was as follows:

The scoring in part of the interview schedule was as follows:

\section{Adoption tool}

Assessment was done on three point continuum as always, sometimes and never with scores of 2, 1 and 0 respectively. 


\section{Measurement of Adoption}

In Agricultural services, adoption of improved practices on various aspects was measured. There was variation in number of aspects, improved practices and scores in each Agriculture service as presented in Table 1.

The respondents were categorized according to MPS in three equal interval categories as high, medium and low adoption, given in Table 2.

\section{Statistical analysis of data}

After collecting data it was necessary to analyze with the help of statistics to derive proper and adequate conclusion. Therefore, obtained data were coded, tabulated and analyzed with the help of statistical tools and techniques. Frequency and percentages were used to analyze knowledge and adoption.

\section{Results and Discussion}

In adoption of each vegetable practice, the recommended practices adopted by the respondents was studied and presented in Tables 3 to 6 . In each service, respondents were categorized in high, medium and low adoption categories along with MPS.

\section{Adoption of vegetable development service}

Vegetable Development service is a bunch of four vegetables i.e. onion, okra, tomato and brinjal hence the adoption of improved practice related to these four vegetables by respondents is presented in Table 3 to 6 .

\section{Adoption of improved onion cultivation practices}

Data presented in Table 3 depicts that in onion cultivation practices, beneficiaries had high extent of adoption in seven components and medium adoption in three components. In the aspect high yielding varieties of onion, beneficiaries had high MPS (82) also reflected in distribution as 70 per cent of the beneficiaries were in high adoption category. Planting through seeds was adopted by all the beneficiaries $(100 \%)$ to high extent with MPS 100. Recommended seed rate was adopted to high extent with MPS 67 and 56.66 per cent beneficiaries were in high adoption category and 20 per cent beneficiaries were in medium adoption category. Adoption of plant spacing in the onion was followed by half of the beneficiaries $(50 \%)$ to high extent followed by one third $(33.33 \%)$ to medium extent also revealed in high MPS (67). In following irrigation schedule, the MPS of beneficiaries was 95 indicating high adoption, as majority of the beneficiaries (90\%) was in high adoption category. Recommended quantity of FYM in onion was adopted to high extent by 66.66 per cent beneficiaries and medium adoption by 13.33 per cent beneficiaries also revealed in high MPS (73). For crop maturity period MPS was 82 reflecting high adoption and the practice was adopted to high extent by 70 per cent beneficiaries.

In rest of the three components viz doses of fertilizers, Insecticides and pesticides, beneficiaries had medium extent of adoption with MPS 63, 47 and 60 respectively. Distribution of beneficiaries also indicate that in fertilizers doses, 46.66 per cent and 33.33 per cent beneficiaries were in high and medium adoption category respectively while in insecticides 40 per cent and 33.33 per cent beneficiaries were in medium and poor adoption categories respectively. In pesticides, 40 per cent beneficiaries were in high as well as medium adoption category.

Table 3 further reveal that non-beneficiaries also had high extent of adoption in two components i.e. in planting method and crop maturity period as reflected by the MPS 100 
and 70 respectively and the distribution of respondents also reveal that 100 per cent and 53.33 per cent non-beneficiaries respectively were in high adoption category. In six components i.e. high yielding varieties, seed rate, plant spacing, irrigation schedule, application of FYM quantity, pesticides nonbeneficiaries had medium extent of adoption as the MPS ranging from 37 - 58. Further category wise distribution of non-beneficiaries according to knowledge categories also reveals that 23.33 - 46.66 per cent nonbeneficiaries were in medium adoption category in these components of onion cultivation. In rest two components of onion cultivation i.e. fertilizers and insecticides, nonbeneficiaries had low extent of adoption with MPS 22 and 30 respectively, also evident from the category wise distribution of nonbeneficiaries i.e. 46.66 and 50 per cent nonbeneficiary respondents were in low adoption category and 33.33 per cent and 40 per cent non-beneficiaries respectively were in medium adoption category in the components of onion cultivation.

\section{Adoption of improved okra cultivation practices}

Perusal of Table 4 reveals that beneficiaries had high extent of adoption in eight components of okra cultivation practices. In the components high yielding varieties of okra, beneficiaries had high MPS (83) and 76.66 per cent of the beneficiaries were in high adoption category. Planting through seeds was adopted completely by all the beneficiaries with MPS 100. High adoption of recommended seed rate by the beneficiaries as reflected with MPS 78, also evident in adoption categories where 63.33 per cent beneficiaries were in high adoption category and 30 per cent beneficiaries were in medium adoption category. Adoption of specific plant spacing in the okra crop was followed to high extent by 60 per cent beneficiaries also revealed in the high MPS - 72. In following correct irrigation schedule, the beneficiaries had high MPS i.e. 92, and majority of the beneficiaries $(83.33 \%)$ were in high adoption category. In application of fertilizers, appropriate dose was adopted by 56.66 per cent beneficiaries to high extent and 26.66 per cent beneficiaries to medium extent reflected in high MPS 70. Farm yard manure was highly adopted by 73.33 per cent beneficiaries also depicted in MPS 82. High MPS (80) for crop maturity period among the beneficiaries also reflected in distribution, as by 66.66 per cent beneficiaries were in the high adoption category and 26.66 per cent beneficiaries were in medium adoption category in the components of okra cultivation.

In rest two components viz use of insecticides (52 MPS) and pesticides (58 MPS) in okra cultivation medium extent of adoption was observed among the beneficiaries of okra cultivation also reflected in distribution of beneficiaries, as in insecticides 33.33, 36.66 and 30 per cent beneficiaries respectively were in high, medium, and low adoption categories. While in case of pesticides 33.33, 50 and 16.66 per cent beneficiaries respectively were in high, medium and low adoption category.

With regard to non-beneficiaries (Table 4), it is important to note that high extent of adoption was found in two components of okra cultivation i.e. in planting method and irrigation schedule as revealed by MPS 100 and 70, respectively and all the nonbeneficiaries (100\%) were in high adoption category in planting method while in irrigation schedule, 56.66 per cent non-beneficiaries were in high and 23.33 per cent nonbeneficiaries were in medium adoption category. In rest eight components of okra cultivation i.e. variety grown, seed rate, spacing kept, doses of fertilizers, application of FYM quantity, insecticides and pesticides, diseases and control measures and crop 
maturity period, non-beneficiaries had medium extent of adoption as the MPS ranges or from 35 - 60. Further category wise distribution of the non-beneficiaries in these components (Table 4), further reveal that 30 60 per cent non-beneficiaries were in medium adoption category of okra cultivation however, 13.33 - 43.33 per cent non-beneficiaries were in high adoption category and 16.66 - 46.66 per cent non-beneficiaries in poor adoption category.

The findings of the present study are in line with Singh et al., (2005) who found that recommended sowing rate was used by 94 per cent of the respondents. More than 25 per cent of the respondents had given first irrigation as recommended; some supplied less or more irrigation. The recommended plant-to-plant spacing was adopted by 20 per cent of the respondents; the others reduced or increased the plant spacing. Approximately 40 per cent maintained the row spacing at $45 \mathrm{~cm}$; the others reduced or increased the row spacing. FYM [farmyard manure] was applied at the recommended rate by 34.67 per cent of the respondents. Some increased or reduced the FYM rate, whereas others did not use FYM.
More than 60 per cent of the farmers conducted hoeing as recommended. Only 10.67 per cent had sprayed pesticides at recommended rates; the rest had applied pesticides at rates higher by 300-600 per cent. Only 17.33 per cent had applied urea during sowing and at 40-45 days after sowing (DAS); some had applied urea at 4-5 and 30-45 DAS, and at 8-10 and 30-45 DAS. Scarcity of labour was identified by 83.33 per cent of the respondents as the main constraint in okra production.

\section{Adoption of improved tomato cultivation practices}

Data in Table 5 regarding over all adoption of tomato cultivation practices high adoption (67.27 MPS) also depicts from distribution of beneficiaries that 53.33 per cent and 46.66 per cent of the beneficiaries were in high and medium adoption categories respectively. Non-beneficiaries were found in medium adoption category with MPS 55 and distribution of beneficiaries reveals that 53.33 per cent and 46.66 per cent of the beneficiaries were in medium and low adoption categories respectively.

Table.1 Service wise details of adoption scale

\begin{tabular}{|c|c|c|c|c|}
\hline $\begin{array}{c}\text { S. } \\
\text { No }\end{array}$ & $\begin{array}{c}\text { Agricultural Services } \\
\text { Number of } \\
\text { Aspects }\end{array}$ & $\begin{array}{c}\text { Number of } \\
\text { practices }\end{array}$ & $\begin{array}{c}\text { Maximum } \\
\text { Score }\end{array}$ \\
\hline 1 & Vegetable Development & 10 & 10 & 20 \\
\hline
\end{tabular}

Table.2 Adoption categories of respondents

\begin{tabular}{|c|c|}
\hline Categories & Score Range \\
\hline High & $66.67-100$ \\
Medium & $33.34-66.66$ \\
Low & $0-33.33$ \\
\hline
\end{tabular}


Table.3 Distribution of the respondents by the adoption of improved onion cultivation practices

\begin{tabular}{|c|c|c|c|c|c|c|c|c|c|}
\hline \multirow{3}{*}{$\begin{array}{l}\text { S. } \\
\text { No }\end{array}$} & \multirow{3}{*}{ Components } & \multirow{2}{*}{\multicolumn{8}{|c|}{ Extent of adoption }} \\
\hline & & & & & & & & & Non - Beneficiaries $n_{2}=30 \mathrm{f}(\%)$ \\
\hline & & High & Medium & Low & MPS & High & Medium & Low & MPS \\
\hline 1 & $\begin{array}{l}\text { High yielding } \\
\text { varieties }\end{array}$ & $\begin{array}{c}21 \\
(70)\end{array}$ & $\begin{array}{c}7 \\
(23.33)\end{array}$ & $\begin{array}{c}2 \\
(6.66)\end{array}$ & 82 & $\begin{array}{l}12 \\
(40)\end{array}$ & $\begin{array}{c}11 \\
(36.66)\end{array}$ & $\begin{array}{c}7 \\
(23.33)\end{array}$ & 58 \\
\hline 2 & Planting methods & $\begin{array}{c}30 \\
(100)\end{array}$ & 0 & 0 & 100 & $\begin{array}{c}30 \\
(100)\end{array}$ & 0 & 0 & 100 \\
\hline 3 & Seed Rate & $\begin{array}{c}17 \\
(56.66)\end{array}$ & $\begin{array}{c}6 \\
(20)\end{array}$ & $\begin{array}{c}7 \\
(23.33)\end{array}$ & 67 & $\begin{array}{c}8 \\
(26.66)\end{array}$ & $\begin{array}{c}11 \\
(36.66)\end{array}$ & $\begin{array}{c}11 \\
(36.66)\end{array}$ & 45 \\
\hline 4 & Spacing & $\begin{array}{c}15 \\
(50)\end{array}$ & $\begin{array}{c}10 \\
(33.33)\end{array}$ & $\begin{array}{c}5 \\
(16.66)\end{array}$ & 67 & $\begin{array}{c}8 \\
(26.66)\end{array}$ & $\begin{array}{c}14 \\
(46.66)\end{array}$ & $\begin{array}{c}8 \\
(26.66)\end{array}$ & 50 \\
\hline 5 & Irrigation Schedule & $\begin{array}{c}27 \\
(90)\end{array}$ & $\begin{array}{c}3 \\
(10)\end{array}$ & 0 & 95 & $\begin{array}{c}18 \\
(60)\end{array}$ & $\begin{array}{c}7 \\
(23.33)\end{array}$ & $\begin{array}{c}5 \\
(16.66)\end{array}$ & 43 \\
\hline 6 & Fertilizers & $\begin{array}{c}14 \\
(46.66)\end{array}$ & $\begin{array}{c}10 \\
(33.33)\end{array}$ & $\begin{array}{c}6 \\
(20)\end{array}$ & 63 & $\begin{array}{c}6 \\
(20)\end{array}$ & $\begin{array}{c}10 \\
(33.33)\end{array}$ & $\begin{array}{c}14 \\
(46.66)\end{array}$ & 22 \\
\hline 7 & FYM & $\begin{array}{c}20 \\
(66.66)\end{array}$ & $\begin{array}{c}4 \\
(13.33)\end{array}$ & $\begin{array}{c}6 \\
(20)\end{array}$ & 73 & $\begin{array}{c}13 \\
(43.33)\end{array}$ & $\begin{array}{c}11 \\
(36.66)\end{array}$ & $\begin{array}{c}6 \\
(20)\end{array}$ & 37 \\
\hline 8 & Insecticides & $\begin{array}{c}8 \\
(26.66)\end{array}$ & $\begin{array}{c}12 \\
(40)\end{array}$ & $\begin{array}{c}10 \\
(33.33)\end{array}$ & 47 & $\begin{array}{c}3 \\
(10)\end{array}$ & $\begin{array}{c}12 \\
(40)\end{array}$ & $\begin{array}{c}15 \\
(50)\end{array}$ & 30 \\
\hline 9 & Pesticides & $\begin{array}{c}12 \\
(40)\end{array}$ & $\begin{array}{c}12 \\
(40)\end{array}$ & $\begin{array}{c}6 \\
(20)\end{array}$ & 60 & $\begin{array}{c}7 \\
(23.33)\end{array}$ & $\begin{array}{c}13 \\
(43.33)\end{array}$ & $\begin{array}{c}10 \\
(33.33)\end{array}$ & 45 \\
\hline 10 & Maturity Period & $\begin{array}{c}21 \\
(70)\end{array}$ & $\begin{array}{c}7 \\
(23.33)\end{array}$ & $\begin{array}{c}2 \\
(6.66)\end{array}$ & 82 & $\begin{array}{c}16 \\
(53.33)\end{array}$ & $\begin{array}{c}10 \\
(33.33)\end{array}$ & $\begin{array}{c}4 \\
(13.33)\end{array}$ & 70 \\
\hline \multicolumn{2}{|c|}{ Over : } & $\begin{array}{c}15 \\
(50)\end{array}$ & $\begin{array}{c}15 \\
(50)\end{array}$ & $\mathbf{0}$ & 72.88 & 0 & $\begin{array}{c}19 \\
(63.33)\end{array}$ & $\begin{array}{c}11 \\
(36.66)\end{array}$ & 37.88 \\
\hline
\end{tabular}

Table.4 Distribution of the respondents by the adoption of improved okra cultivation practices

\begin{tabular}{|c|c|c|c|c|c|c|c|c|c|}
\hline \multirow{4}{*}{ S. No } & \multirow{4}{*}{ Components } & & & & & & & & 60 \\
\hline & & \multicolumn{8}{|c|}{ Extent of adoption } \\
\hline & & \multicolumn{4}{|c|}{ Beneficiaries $n_{1}=30 \mathrm{f}(\%)$} & \multicolumn{4}{|c|}{ Non - Beneficiaries $n_{2}=30 f(\%)$} \\
\hline & & High & Medium & Low & MPS & High & Medium & Low & MPS \\
\hline 1 & High yielding varieties & $\begin{array}{c}23 \\
(76.66)\end{array}$ & $\begin{array}{c}4 \\
(13.33)\end{array}$ & $\begin{array}{c}3 \\
(10)\end{array}$ & 83 & $\begin{array}{c}12 \\
(40)\end{array}$ & $\begin{array}{c}9 \\
(30)\end{array}$ & $\begin{array}{c}9 \\
(30)\end{array}$ & 55 \\
\hline 2 & Planting methods & $\begin{array}{c}30 \\
(100)\end{array}$ & 0 & 0 & 100 & $\begin{array}{c}30 \\
(100)\end{array}$ & 0 & 0 & 100 \\
\hline 3 & Seed Rate & $\begin{array}{c}19 \\
(63.33)\end{array}$ & $\begin{array}{c}9 \\
(30)\end{array}$ & $\begin{array}{c}2 \\
(6.66)\end{array}$ & 78 & $\begin{array}{c}11 \\
(36.66)\end{array}$ & $\begin{array}{c}9 \\
(30)\end{array}$ & $\begin{array}{c}10 \\
(33.33)\end{array}$ & 52 \\
\hline 4 & Spacing & $\begin{array}{c}18 \\
(60)\end{array}$ & $\begin{array}{c}7 \\
((23.33)\end{array}$ & $\begin{array}{c}5 \\
(16.66)\end{array}$ & 72 & $\begin{array}{c}9 \\
(30)\end{array}$ & $\begin{array}{c}10 \\
(33.33)\end{array}$ & $\begin{array}{c}11 \\
(36.66)\end{array}$ & 47 \\
\hline 5 & Irrigation Schedule & $\begin{array}{c}25 \\
(83.33)\end{array}$ & $\begin{array}{c}5 \\
(16.66)\end{array}$ & 0 & 92 & $\begin{array}{c}17 \\
(56.66)\end{array}$ & $\begin{array}{c}7 \\
(23.33)\end{array}$ & $\begin{array}{c}6 \\
(20)\end{array}$ & 70 \\
\hline 6 & Fertilizers & $\begin{array}{c}17 \\
(56.66)\end{array}$ & $\begin{array}{c}8 \\
(26.66)\end{array}$ & $\begin{array}{c}5 \\
(16.66)\end{array}$ & 70 & $\begin{array}{c}5 \\
(16.66)\end{array}$ & $\begin{array}{c}11 \\
(36.66)\end{array}$ & $\begin{array}{c}14 \\
(46.66)\end{array}$ & 35 \\
\hline 7 & FYM & $\begin{array}{c}22 \\
(73.33)\end{array}$ & $\begin{array}{c}5 \\
(16.66)\end{array}$ & $\begin{array}{c}3 \\
(10)\end{array}$ & 82 & $\begin{array}{c}13 \\
(43.33)\end{array}$ & $\begin{array}{c}10 \\
(33.33)\end{array}$ & $\begin{array}{c}7 \\
(23.33)\end{array}$ & 60 \\
\hline 8 & Insecticides & $\begin{array}{c}10 \\
(33.33)\end{array}$ & $\begin{array}{c}11 \\
(36.66)\end{array}$ & $\begin{array}{c}9 \\
(30)\end{array}$ & 52 & $\begin{array}{c}7 \\
(23.33)\end{array}$ & $\begin{array}{c}15 \\
(50)\end{array}$ & $\begin{array}{c}8 \\
(26.66)\end{array}$ & 48 \\
\hline 9 & Pesticides & $\begin{array}{c}10 \\
(33.33)\end{array}$ & $\begin{array}{c}15 \\
(50)\end{array}$ & $\begin{array}{c}5 \\
(16.66)\end{array}$ & 58 & $\begin{array}{c}5 \\
(16.66)\end{array}$ & $\begin{array}{c}18 \\
(60)\end{array}$ & $\begin{array}{c}7 \\
(23.33)\end{array}$ & 47 \\
\hline 10 & Maturity Period & $\begin{array}{c}20 \\
(66.66)\end{array}$ & $\begin{array}{c}8 \\
(26.66)\end{array}$ & $\begin{array}{c}2 \\
(6.66)\end{array}$ & 80 & $\begin{array}{c}12 \\
(40)\end{array}$ & $\begin{array}{c}13 \\
(43.33)\end{array}$ & $\begin{array}{c}5 \\
(16.66)\end{array}$ & 62 \\
\hline & Over all & $\begin{array}{c}17 \\
(56.66)\end{array}$ & $\begin{array}{c}11 \\
(36.66)\end{array}$ & $\begin{array}{c}2 \\
(6.66)\end{array}$ & 77.27 & $\begin{array}{c}3 \\
(10)\end{array}$ & $\begin{array}{c}24 \\
(80)\end{array}$ & $\begin{array}{c}3 \\
(10)\end{array}$ & 52.12 \\
\hline
\end{tabular}


Table.5 Distribution of the respondents by the adoption of improved tomato cultivation Practices

\begin{tabular}{|c|c|c|c|c|c|c|c|c|c|}
\hline \multirow[t]{3}{*}{ S. No } & \multirow[t]{3}{*}{ Components } & \multicolumn{8}{|c|}{ Extent of adoption } \\
\hline & & \multicolumn{4}{|c|}{ Beneficiaries $\mathrm{n}_{1}=\mathbf{3 0} \mathrm{f}(\%)$} & \multicolumn{4}{|c|}{ Non - Beneficiaries $\mathbf{n}_{2}=30 \mathrm{f}(\%)$} \\
\hline & & High & Medium & Low & MPS & High & Medium & Low & MPS \\
\hline 1 & High yielding varieties & $\begin{array}{c}21 \\
(70)\end{array}$ & $\begin{array}{c}5 \\
(16.66)\end{array}$ & $\begin{array}{c}4 \\
(13.33)\end{array}$ & 78 & $\begin{array}{c}12 \\
(40)\end{array}$ & $\begin{array}{c}13 \\
(43.33)\end{array}$ & $\begin{array}{c}5 \\
(16.66)\end{array}$ & 62 \\
\hline 2 & Planting methods & $\begin{array}{c}30 \\
(100)\end{array}$ & 0 & 0 & 100 & $\begin{array}{c}30 \\
(100)\end{array}$ & 0 & 0 & 100 \\
\hline 3 & Seed Rate & $\begin{array}{c}16 \\
(53.33)\end{array}$ & $\begin{array}{c}5 \\
(16.66)\end{array}$ & $\begin{array}{c}9 \\
(30)\end{array}$ & 62 & $\begin{array}{c}11 \\
(36.66)\end{array}$ & $\begin{array}{c}10 \\
(33.33)\end{array}$ & $\begin{array}{c}9 \\
(30)\end{array}$ & 55 \\
\hline 4 & Plant Spacing & $\begin{array}{c}12 \\
(40)\end{array}$ & $\begin{array}{c}14 \\
(46.66)\end{array}$ & $\begin{array}{c}4 \\
(13.33)\end{array}$ & 63 & $\begin{array}{c}6 \\
(20)\end{array}$ & $\begin{array}{c}16 \\
(53.33)\end{array}$ & $\begin{array}{c}8 \\
(26.66)\end{array}$ & 47 \\
\hline 5 & Irrigation Schedule & $\begin{array}{c}22 \\
(76.66)\end{array}$ & $\begin{array}{c}8 \\
(26.66)\end{array}$ & 0 & 87 & $\begin{array}{c}13 \\
(43.33)\end{array}$ & $\begin{array}{c}11 \\
(36.66)\end{array}$ & $\begin{array}{c}6 \\
(20)\end{array}$ & 62 \\
\hline 6 & Fertilizers & $\begin{array}{c}19 \\
(63.33)\end{array}$ & $\begin{array}{c}9 \\
(30)\end{array}$ & $\begin{array}{c}2 \\
(6.66)\end{array}$ & 78 & $\begin{array}{c}11 \\
(33.33)\end{array}$ & $\begin{array}{c}15 \\
(50)\end{array}$ & $\begin{array}{c}4 \\
(13.33)\end{array}$ & 62 \\
\hline 7 & FYM & $\begin{array}{c}18 \\
(60)\end{array}$ & $\begin{array}{c}12 \\
(40)\end{array}$ & 0 & 80 & $\begin{array}{c}14 \\
(46.66)\end{array}$ & $\begin{array}{c}15 \\
(50)\end{array}$ & $\begin{array}{c}1 \\
(3.33)\end{array}$ & 71.7 \\
\hline 8 & Insecticides & $\begin{array}{c}12 \\
(40)\end{array}$ & $\begin{array}{c}9 \\
(30)\end{array}$ & $\begin{array}{c}9 \\
(30)\end{array}$ & 55 & $\begin{array}{c}5 \\
(16.66)\end{array}$ & $\begin{array}{c}15 \\
(50)\end{array}$ & $\begin{array}{c}10 \\
(33.33)\end{array}$ & 58 \\
\hline 9 & Pesticides & $\begin{array}{c}9 \\
(30)\end{array}$ & $\begin{array}{c}16 \\
(53.33)\end{array}$ & $\begin{array}{c}5 \\
(16.66)\end{array}$ & 57 & $\begin{array}{c}4 \\
(13.33)\end{array}$ & $\begin{array}{c}17 \\
(56.66)\end{array}$ & $\begin{array}{c}9 \\
(30)\end{array}$ & 42 \\
\hline 10 & Maturity Period & $\begin{array}{c}19 \\
(63.33)\end{array}$ & $\begin{array}{c}10 \\
(33.33)\end{array}$ & $\begin{array}{c}1 \\
(3.33)\end{array}$ & 80 & $\begin{array}{c}12 \\
(40)\end{array}$ & $\begin{array}{c}12 \\
(40)\end{array}$ & $\begin{array}{c}6 \\
(20)\end{array}$ & 60 \\
\hline Over all & & $\begin{array}{c}16 \\
(53.33)\end{array}$ & $\begin{array}{c}14 \\
(46.66)\end{array}$ & $\mathbf{0}$ & 67.27 & $\mathbf{0}$ & $\begin{array}{c}16 \\
(53.33)\end{array}$ & $\begin{array}{c}14 \\
(46.66)\end{array}$ & 55 \\
\hline
\end{tabular}

Table.6 Distribution of the respondents by the adoption of improved brinjal cultivation practices

\begin{tabular}{|c|c|c|c|c|c|c|c|c|c|}
\hline \multirow[t]{3}{*}{ S. No } & \multirow[t]{3}{*}{ Components } & \multicolumn{8}{|c|}{ Extent of Adoption } \\
\hline & & \multicolumn{4}{|c|}{ Beneficiaries $\mathbf{n}_{1}=30 \mathrm{f}(\%)$} & \multicolumn{4}{|c|}{ Non - Beneficiaries $n_{2}=30 f(\%)$} \\
\hline & & High & Medium & Low & MPS & High & Medium & Low & MPS \\
\hline 1 & $\begin{array}{l}\text { High yielding } \\
\text { varieties }\end{array}$ & $\begin{array}{c}19 \\
(63.33)\end{array}$ & $\begin{array}{c}9 \\
(30)\end{array}$ & $\begin{array}{c}2 \\
(6.66)\end{array}$ & 78.33 & $\begin{array}{c}11 \\
(36.66)\end{array}$ & $\begin{array}{c}13 \\
(43.33)\end{array}$ & $\begin{array}{c}6 \\
(20)\end{array}$ & $\mathbf{5 8 . 3}$ \\
\hline 2 & $\begin{array}{l}\text { Planting } \\
\text { methods }\end{array}$ & $\begin{array}{c}30 \\
(100)\end{array}$ & 0 & 0 & 100 & $\begin{array}{c}30 \\
(100)\end{array}$ & 0 & 0 & 100 \\
\hline 3 & Seed Rate & $\begin{array}{l}21 \\
(70)\end{array}$ & $\begin{array}{c}7 \\
(23.33)\end{array}$ & $\begin{array}{c}2 \\
(6.66)\end{array}$ & 81.67 & $\begin{array}{c}13 \\
(43.33)\end{array}$ & $\begin{array}{c}13 \\
(43.33)\end{array}$ & $\begin{array}{c}4 \\
(13.33)\end{array}$ & 65 \\
\hline 4 & Plant Spacing & $\begin{array}{c}17 \\
(56.66)\end{array}$ & $\begin{array}{c}11 \\
(36.66)\end{array}$ & $\begin{array}{c}2 \\
(6.66)\end{array}$ & 75 & $\begin{array}{c}9 \\
(30)\end{array}$ & $\begin{array}{c}16 \\
(53.33)\end{array}$ & $\begin{array}{c}5 \\
(16.66)\end{array}$ & 56.7 \\
\hline 5 & $\begin{array}{l}\text { Irrigation } \\
\text { Schedule }\end{array}$ & $\begin{array}{c}16 \\
(53.33)\end{array}$ & $\begin{array}{c}5 \\
(16.66)\end{array}$ & $\begin{array}{c}9 \\
(30)\end{array}$ & 62 & $\begin{array}{c}12 \\
(40)\end{array}$ & $\begin{array}{c}10 \\
(33.33)\end{array}$ & $\begin{array}{c}8 \\
(26.66)\end{array}$ & 57 \\
\hline 6 & Fertilizers & $\begin{array}{c}16 \\
(53.33)\end{array}$ & $\begin{array}{c}5 \\
(16.66)\end{array}$ & $\begin{array}{c}9 \\
(30)\end{array}$ & 62 & $\begin{array}{c}7 \\
(23.33)\end{array}$ & $\begin{array}{c}10 \\
(33.33)\end{array}$ & $\begin{array}{c}13 \\
(43.33)\end{array}$ & 40 \\
\hline 7 & FYM & $\begin{array}{c}22 \\
(73.33)\end{array}$ & $\begin{array}{c}8 \\
(26.66)\end{array}$ & 0 & 87 & $\begin{array}{c}16 \\
(53.33)\end{array}$ & $\begin{array}{c}11 \\
(36.66)\end{array}$ & $\begin{array}{c}3 \\
(10)\end{array}$ & 72 \\
\hline 8 & Insecticides & $\begin{array}{c}14 \\
(46.66)\end{array}$ & $\begin{array}{c}10 \\
(33.33)\end{array}$ & $\begin{array}{c}6 \\
(20)\end{array}$ & 63 & $\begin{array}{c}8 \\
(26.66)\end{array}$ & $\begin{array}{c}14 \\
(46.66)\end{array}$ & $\begin{array}{c}8 \\
(26.66)\end{array}$ & 50 \\
\hline 9 & Pesticides & $\begin{array}{c}13 \\
(43.33)\end{array}$ & $\begin{array}{c}9 \\
(30)\end{array}$ & $\begin{array}{c}8 \\
(26.66)\end{array}$ & 58 & $\begin{array}{c}6 \\
(20)\end{array}$ & $\begin{array}{c}15 \\
(50)\end{array}$ & $\begin{array}{c}9 \\
(30)\end{array}$ & 45 \\
\hline 10 & Maturity Period & $\begin{array}{c}20 \\
(66.66)\end{array}$ & $\begin{array}{c}7 \\
(23.33)\end{array}$ & $\begin{array}{c}3 \\
(10)\end{array}$ & 78 & $\begin{array}{c}13 \\
(43.33)\end{array}$ & $\begin{array}{c}12 \\
(40)\end{array}$ & $\begin{array}{c}5 \\
(16.66)\end{array}$ & 63 \\
\hline & Over all & $\begin{array}{c}16 \\
(53.33)\end{array}$ & $\begin{array}{c}14 \\
(46.66)\end{array}$ & $\mathbf{0}$ & 67.72 & $\begin{array}{c}3 \\
(10)\end{array}$ & $\begin{array}{l}15 \\
(50)\end{array}$ & $\begin{array}{c}12 \\
(40)\end{array}$ & 55.30 \\
\hline
\end{tabular}


A glance over the table depict that beneficiaries had high extent of adoption in seven components as compared to non-beneficiaries who had high extent of adoption in two components only.

Regarding beneficiaries adoption of tomato cultivation practices, it can be seen that high MPS i.e. 78 in recommended tomato varieties also revealed by 70 per cent beneficiaries falling in high adoption category. Further planting through seeds was adopted by all the beneficiaries to high extent also reflected by MPS 100. Recommended irrigation schedule was adopted in tomato cultivation by 73.33 per cent beneficiaries to high extent also depicted in high MPS (87). Advised doses of fertilizers in tomato cultivation were adopted by 63.33 per cent beneficiaries to high extent and medium extent by 30 per cent beneficiaries, also revealed by high MPS (78). MPS (80) was calculated for FYM also revealed by the adoption categories as 60 per cent beneficiaries were in high extent and 40 per cent beneficiaries in medium extent categories. Maturity period of tomato crop was highly adopted by 63.33 per cent beneficiaries and 33.33 per cent beneficiaries were in medium adoption category and the MPS 80 for the practice also indicates high extent of adoption. In rest four practices, medium extent of adoption was found i.e. recommended seed rate was adopted by 53.33 per cent to high extent and 16.66 per cent beneficiaries to medium extent with medium MPS 62. The practices related to plant spacing were adopted by 40 per cent and 46.66 per cent beneficiaries to high and medium extent respectively as also evident by medium MPS - 63. Insecticides were adopted by 40 per cent and 30 per cent beneficiaries to high and medium extent according to adoption categories respectively and also 30 per cent beneficiaries in low adoption categories leading to medium extent of adoption also evident in MPS (55). Pesticides were adopted by 53.33 per cent beneficiaries to medium extent and 30 per cent beneficiaries to high extent and 16.66 per cent beneficiaries also in low adoption category leading to medium extent of adoption with MPS - 57. In case of non-beneficiaries, Table 5 further reveals that high extent of adoption was found in two components i.e., planting method and quantity of FYM application with MPS 100 and 71.7 respectively and were falling in high (100 and 46.66) and medium (50) adoption categories respectively. In rest nine components nonbeneficiaries had medium adoption viz high yeiding varieties (62 MPS), seed rate (55 MPS), plant spacing (47 MPS), irrigation schedule (62 MPS), fertilizers (62 MPS), insecticides (58 MPS), pesticides (42 MPS) and crop maturity period (60 MPS) as indicated by MPS which is also revealed in category wise distribution as 33.33 - 56.66 per cent non-beneficiaries were in medium adoption category and 13.33 - 43.33 per cent non-beneficiaries were in low adoption category of the tomato cultivation practices.

The findings are in conformity with Kumar et al., (2004) that high majority of the farmers cultivated tomato hybrids, planted during JuneJuly, followed 75-100 g per ha seed rate, adopted $60 \times 40 \mathrm{~cm}$ spacing and applied only 510 tones of FYM per ha.

\section{Adoption of improved brinjal cultivation practices}

In Vegetable Development service, data related to Brinjal cultivation practices is presented in Table 6. The overall adoption of beneficiaries in brinjal cultivation was high as depicted by MPS 67.72 and 53.33 percent beneficiaries were falling in high adoption category and 46.66 percent beneficiaries were falling in medium adoption category. In case of non-beneficiaries, MPS 55.30 indicates medium adoption and 50 percent and 40 per cent of the non-beneficiaries were falling in medium and low adoption categories of Brinjal cultivation respectively.

Further the table depicts that in brinjal cultivation, beneficiaries had high extent of adoption in six practices. In these components, high yielding varieties of brinjal, beneficiaries had high MPS 78.33 also reflected in distribution as 63.33 per cent of the 
beneficiaries were in high adoption category. Planting through seeds was adapted to high extent as all the beneficiaries were in high adoption category and MPS was also100. Recommended seed rate was adapted to high extent by 70 per cent beneficiaries and medium extent by 23.33 per cent beneficiaries also evident by high MPS (81.67). Adoption of plant spacing in the brinjal crop was followed by 56.66 per cent beneficiaries to high extent also revealed by high MPS (75). Recommended quantity of FYM in brinjal was highly adopted by 76.66 per cent beneficiaries and 26.66 per cent beneficiaries were in medium adoption category also revealed in MPS - 87. MPS for crop maturity period was found to be high as 78 and the practice was also adopted to high extent by 66.66 per cent beneficiaries.

In rest four components viz., irrigation schedule, doses of fertilizers, Insecticides and pesticides beneficiaries had medium extent of adoption with MPS 62, 62, 63 and 58 respectively and $16.66-33.33$ per cent of the beneficiaries were falling in medium and 20 30 per cent of the beneficiaries were in low adoption category.

Table 6 further reveals that non-beneficiaries also had high extent of adoption in two components i.e. in planting method and FYM with MPS 100 and 72 respectively. In rest of the eight components i.e. improved varieties, seed rate, plant spacing, irrigation schedule, application of FYM quantity and diseases and control measures, non-beneficiaries had medium extent of adoption as the MPS ranging between 40 - 65. Further category wise distribution also reveals that 33.33 - 53.33 per cent non-beneficiaries were in medium adoption category in these practices of brinjal cultivation. In adoption of the programme, majority of the beneficiaries were in high and medium adoption categories in various components of all the programme and few of the beneficiaries were in low adoption category while in case of nonbeneficiaries all were in medium or low adoption categories in the vegetable development programme.

\section{References}

Dave, R., Godawat, A and Soni, R.L. 2011. Adoption of okra production technologies in tribal women promoted under NAIP. Rajasthan journal of Extension Education 19: 117-125.

Devi1, U. S., Ram, D. and Vishram, R. 2013. Adoption of IPM by Cabbage and Cauliflower Growers in Manipur. Journal of Community Mobilization and Sustainable Development 8: 218-222.

Nath, D. and Biswas, P. K. 2011. Production Constraints of Vegetable Cultivation in West Tripura. Journal of Community Mobilization and Sustainable Development 6: 177-179.

Samantaray, S.K, Prusty, S and Raj, R. K. 2009. Constraints in vegetable Production Experience of Tribal Vegetable Growers. Indian Research Journal of Extension Education 9: 32-34.

Sharma, M and Ladher, S. 2010. Constraints in the farm practices of major vegetable crops. Kisan World 37: 23-24.

Singh, G. and Agrawal, R.P. 2013. Impact of Front Line Demonstration of Improved Okra Cultivation Technology. Journal of Community Mobilization and Sustainable Development 8: 291-293.

\section{How to cite this article:}

Sonali Sharma, Snehlata Maheshwari, Rajshree Upadhyay, Bhupendra Upadhyay and Morwal, B.R. 2018. Adoption of Improved Practices Promoted under Vegetable Development Programme among the Tribals. Int.J.Curr.Microbiol.App.Sci. 7(08): 4040-4048. doi: https://doi.org/10.20546/ijcmas.2018.708.419 